\title{
Erratum to: Serum albumin, a good indicator of persistent organ failure in acute pancreatitis
}

\author{
Shoukang $\mathrm{Li}^{1+}$, Yushun Zhang ${ }^{1 \dagger}$, Mengjiao $\mathrm{Li}^{2}$, Chao Xie ${ }^{1}$ and Heshui $\mathrm{Wu}^{1 *}$
}

\section{Erratum}

Following publication of this article [1], it has come to our attention that the affiliations were captured as:

1 Pancreatic Disease Institute, Union Hospital, Tongji Medical College, Huazhong University of Science and Technology, 430022 Wuhan, Hubei Province, People's Republic of China. 2 Department of Endocrinology, Union Hospital, Tongji Medical College, Huazhong University of Science and Technology, 430022 Wuhan, Hubei Province, People's Republic of China.

However, the correct affiliations should be as following:

1 Department of Pancreatic Surgery, Union Hospital, Tongji Medical College, Huazhong University of Science and Technology, Wuhan 430022, China.

2 Department of Endocrinology, Union Hospital, Tongji Medical College, Huazhong University of Science and Technology, Wuhan 430022, China.

\footnotetext{
Author details

'Department of Pancreatic Surgery, Union Hospital, Tongji Medical College, Huazhong University of Science and Technology, Wuhan 430022, China.

${ }^{2}$ Department of Endocrinology, Union Hospital, Tongji Medical College, Huazhong University of Science and Technology, Wuhan 430022, China.
}

Received: 26 June 2017 Accepted: 4 July 2017

Published online: 07 July 2017

\section{Reference}

1. Li S, Zhang Y, Li M, Xie C, Wu H. Serum albumin, a good indicator of persistent organ failure in acute pancreatitis. BMC Gastroenterol. 2017;17:59. doi:10.1186/s12876-017-0615-8.

\footnotetext{
* Correspondence: heshuiwu@hust.edu.cn

${ }^{\dagger}$ Equal contributors

'Department of Pancreatic Surgery, Union Hospital, Tongji Medical College,

Huazhong University of Science and Technology, Wuhan 430022, China
} 